\title{
Conditionally replicating herpes vectors for cancer therapy
}

\author{
Robert L. Martuza \\ Harvard Medical School, Massachusetts General Hospital, WHT502 55 Fruit Street, \\ Boston, Massachusetts 02114, USA. Phone: (617) 726-8581; Fax: (617) 726-4814; E-mail: rmartuza@partners.org.
}

The use of genetically engineered viral vectors for tumor therapy initially involved replication-defective vectors, generally derived from retro- or adenoviruses, for the transfer of genes to cause tumor growth inhibition or tumor destruction. However, because of limitations involving vector delivery and relatively low levels of gene transfer, such vectors systems have proven relatively inefficient for the treatment of large solid tumors. In an attempt to overcome this problem, vectors have been developed that can replicate within the tumor and thus increase the efficiency of tumor cell destruction, but appropriate tumor targeting has been a major challenge for such systems. Vectors are needed that replicate within tumor cells and cause tumor cell destruction but that cause no local or systemic toxicity, because they fail to grow within normal tissues. Studies that I and my colleagues performed initially focused on the treatment of malignant brain tumors using local vector delivery to avoid damaging normal brain and associated tissues. We and others have since expanded the scope of this project to target cancers in the nervous system and elsewhere, using targeted or systemically delivered vectors derived from herpes simplex virus-1 (HSV-1) .

\section{The selection of HSV-1 as a vector system for brain tumor therapy}

As this Perspective series documents, there are many potential replicating vectors worthy of study as oncolytic agents. Our initial interest in HSV-1 as a biotherapeutic agent for cancer derived from the fact that our laboratory focused on neuro-oncology, and herpes simplex viruses were known to be able to replicate within nervous system tissue. Wild-type HSV-1 can cause a destructive encephalitis, but by the time we began these studies, the genome of HSV-1 had been sequenced, and many of the genes associated with neurovirulence had been identified. We anticipated that suitably modified HSV-1 could be applied safely to tumors in the brain.

HSV-1 has many attractive features for development as an oncolytic and gene transfer vector. First, it is large and well-studied. HSV-1 is an enveloped virus containing approximately $152 \mathrm{~kb}$ of DNA. The essential and nonessential genes have been identified, and HSV genetic-engineering techniques have been developed (1). Theoretically, up to $30 \mathrm{~kb}$ of the genome can be replaced with foreign DNA yet still leave a virus capable of replicating in an appropriate cellular environment. Second, multiple genes associated with neurovirulence (1-3) can be deleted without affecting the virus' capacity to replicate within and destroy tumor cells during the normal lytic phase. Third, anti-herpetic agents are available and provide a safety mechanism in case undesired local or systemic infection occurs. Indeed, HSV-1 vectors can be made with increased sensitivity to such agents. Finally, HSV-1 does not integrate into the cellular genome (1), so it cannot cause insertional mutagenesis. Even during viral latency, HSV-1 persists in neurons as an episome.

HSV-1 is organized into unique long $\left(U_{L}\right)$ and unique short $\left(U_{S}\right)$ sequences flanked by inverted repeat sequences $(R)$ (Figure 1). Its genes can be categorized into three groups according to their temporal expression following viral entry into the cell (1): (a) immediate-early (IE or $\alpha)$ genes in general are responsible for regulatory functions and for the initiation of expression of the next set of viral genes. ICP4, discussed below, is one such immediate-early gene; (b) early $(E$ or $\beta)$ genes are next expressed and encode enzymes necessary for DNA replication, such as thymidine kinase (TK; encoded by $\left.U_{L} 23\right)$ and ribonucleotide reductase (RR; encoded by $\left.U_{L} 39-40\right) 3$.); and (c) late ( $L$ or $\gamma$ ) genes encode structural proteins, surface glycoproteins, and other proteins that are important for viral entry, viral egress, cell-to-cell spread, antiviral immune responses, and virus-host interactions. The $\gamma_{1} 34.5$ gene, which occurs in 2 copies in HSV-1 and encodes the protein ICP34.5, is one example discussed in this article.

\section{Targeting HSV replication to cancer cells}

Thymidine kinase is essential for the replication of the DNA virus, and $U_{L} 23$-negative mutants of HSV-1 show decreased neurovirulence. We studied the $U_{L} 23$-negative strain $d l s p t k$ (4) in hopes of targeting actively mitotic tumor cells, which upregulate their endogenous TK and so may bypass the virus' requirement for $U_{L} 23$. Even at very low multiplicity of infection, $d l s p t k$ could replicate within cultured human malignant glioma cells, spread from cell to cell, and effectively destroy a tumor cell monolayer within a week. Moreover, when we inoculated $d l$ sptk directly into human gliomas growing in the brains of athymic mice, we observed dose-dependent tumor cell destruction and increased animal survival; some of the animals treated were fully cured of the cancer (4). In separate studies, we demonstrated that heat-inactivated virus was ineffective, suggesting that lytic viral replication was necessary. Unfortunately, however, further work in this mouse model showed that high titers of $d$ lsptk caused neurotoxicity. Furthermore, because it lacks TK activity, this strain is insensitive to 2 of the most potent anti-herpetic agents, acyclovir and ganciclovir. We opted not to pursue clinical trials of $d l s p t k$, for fear that any untoward viral replication caused by the treatment might prove difficult to contain. 


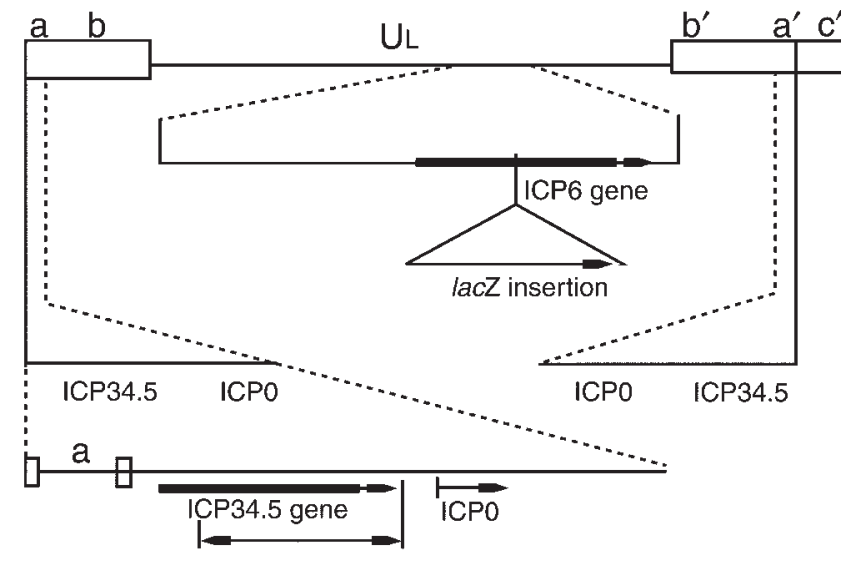

$1.0 \mathrm{~kb}$ deletion in ICP34.5 gene

Because of this concern, we turned our attention to mutations in the $\gamma_{1} 34.5$ gene (5), whose product, ICP34.5, promotes infection of nondividing cells and inhibits apoptosis by infected cells $(1,3)$. Null mutants of $\gamma_{1} 34.5$ do not replicate within healthy adult neurons or cause encephalitis, but grow in permissive, actively cycling cells. Crucially, HSV-1 mutants deficient for ICP34.5 retain their sensitivity to acyclovir and ganciclovir and can destroy malignant glioma cells in culture and in vivo. This mutation would therefore appear to preserve the oncolytic activity of the virus while abolishing its toxicity, but the possibility remained that secondary viral or cellular mutations could suppress its effects. Seeking an additional mutation that could be engineered into ICP34.5-deficient strains to provide still greater assurance of safety, we chose to study hrR3 (2). This strain of HSV1 carries a mutation in the $U_{L} 39$ gene, which encodes the large subunit of HSV ribonucleotide reductase (RR). As with TK, mammalian RR is elevated in tumor cells, so hrR3 might be predicted to grow preferentially within tumor cells. Further, RR-negative mutants had been shown to have decreased neurovirulence and increased sensitivity to acyclovir and ganciclovir, and 2 studies independently confirmed the efficacy of RR-negative mutants in treating malignant glioma $(6,7)$.

\section{Development of a multiple deletion HSV-1 mutant}

We hypothesized that an HSV strain bearing mutations in the genes for ICP34.5 and RR would be safe and effective as a treatment for multiple tumor types (8). We therefore developed G207, a derivative of wildtype HSV-1 strain F in which both $\gamma_{1} 34.5$ genes are deleted and a lac $Z$ insertion inactivates $U_{L} 39$ (Figure 1). Reversion at one of the loci still would leave a vector that was markedly attenuated for neurovirulence, and because the mutations are widely spaced, mutations that could revert more than one locus would not be expected to occur. G207 can be grown to titers in excess of $10^{9}$ plaque forming units ( $\mathrm{pfu}$ ) per $\mathrm{ml}$ using standard techniques, can kill many types of tumor cells in culture, and is hypersensitive to acyclovir and ganciclovir, an attractive safety feature for an initial clinical trial. We therefore proceeded to perform extensive safety testing of G207 (9).

\section{Figure 1}

Targeting HSV to general cancer cell pathways: A general map of HSV-1 demonstrates the unique long (UL) and unique short (US) sequences of HSV-1 flanked by the long and short repeat sequences. Also shown are the mutations introduced to create the vector G207, which infects and lyses tumor cells efficiently but is not virulent in normal tissue, even the brain.

Unlike some other potential biotherapeutic agents, HSV-1 can grow in cells and animals of various species. Indeed, animal models of HSV-1 toxicity are well-developed and include some primate models that are more sensitive than humans to neurotoxicity and death from HSV-1 encephalitis. The safety of G207 has been demonstrated following direct inoculation of up to $10^{7}$ pfu into HSV-sensitive mice by multiple routes and, at doses up to $10^{9} \mathrm{pfu}$, into aotus nancymai, a primate species that is exquisitely sensitive to HSV (9). G207 is now in phase I clinical trial for use in recurrent malignant glioma, and preliminary data (10) suggest that direct intracranial inoculation of G207 at doses up to $3 \times 10^{9}$ pfu causes neither acute toxicity, viral shedding, nor clinically evident delayed reactivation of the virus. Thus, it appears that herpes vectors can be designed to kill cancer cells selectively and can be rationally developed for clinical trial. Indeed, in parallel with our work with G207, multiple laboratories worldwide have explored HSV-1 and HSV-1/HSV-2 hybrids for tumor therapy. One of the more extensively studied vectors is 1716, an ICP34.5-null mutant strain that is effective in killing multiple tumor cell types, although it exhibits some toxicity under certain conditions in animal models (11). Preliminary results of a Phase I study of 1716 in patients with recurrent glioblastoma have also been reported (12). We and others have also explored the use of conditionally replicating HSV-based oncolytic vectors for non-nervous system cancers. Evidence from multiple laboratories shows that such vectors are effective in experimental models of breast (13), prostate (14), colon (15), and ovarian cancers (16) as well as in melanoma (17), head and neck squamous cell cancer (18), and neuroblastoma $(19,20)$.

\section{Using HSV vectors in conjunction with established cancer therapies}

Because conditionally replicating HSV vectors kill tumor cells through pathways that are different from other anticancer therapies, it is reasonable to explore the interactions with other commonly used anti-neoplastic agents. In one study of G207, intraneoplastic inoculation of head and neck squamous cell tumors caused all tumors to regress initially, but tumors recurred in $>50 \%$ of animals. Likewise, cisplatin is a common agent used with variable success to treat this form of cancer. Because cisplatin at physiologically relevant doses does not appear to inhibit G207 replication in cultured cells, the combination of these therapeutics seems promising. Using a tumor cell line that is moderately sensitive to cisplatin, Chalavi et al. (18) showed that a cure rate of $<15 \%$ with cisplatin alone 
or < $50 \%$ with G207 alone could be increased to $100 \%$ when cisplatin and G207 were used in combination.

Radiation therapy is also commonly used for the treatment of solid neoplasms. Investigations of the interactions of radiotherapy with HSV oncolytic vector therapy have only recently been reported. Studies of prostate cancer demonstrated that tumors recurring after irradiation do not develop resistance to HSV-therapy (14). As with cisplatin treatment, irradiation does not appear to decrease viral efficacy, and additive or even synergistic effects are seen in appropriate circumstances $(14,21,22)$.

\section{Immune aspects of HSV tumor therapy}

The oncolytic aspects of conditionally replicating HSVderived vectors, emphasized in the preceding discussion, are sufficient to cause impressive cell killing independent of any interactions with the immune system. Indeed, since most of the early studies were conducted in cell culture or in athymic or SCID mice, the role of the immune system was not explored. More recently, however, studies in syngeneic models have suggested important new avenues for HSV-vector development, exploiting the immune response to HSV-infected tumor cells.

In people, viral infections do not occur in isolation. Various host-virus interactions are important and the roles played by the host immune system are particularly critical. One might imagine that either a pre-existing or a therapeutically elicited immune response to the virus would limit viral replication, eliminate virus, and minimize the therapeutic anti-neoplastic response of an oncolytic HSV. Conversely, one could also imagine that an elicited immune response, if appropriately directed toward tumor cells, might enhance the efficacy of viral tumor therapy. While studies in this area are in their early stages, there is evidence for both of these scenarios. Thus, at least two groups have explored the role of the immune status during therapy, using different syngeneic tumor models in rodents, and have reported that preexisting immunity to HSV influences viral infection of tumor cells, gene expression, and tumor cell destruction. Using a glioma model in syngeneic rats, Herrlinger et al. (23) found that preexisting immunity to HSV reduced expression of a marker gene contained in the vector and increased inflammatory cell responses within intraneoplastically treated tumors. However, in a separate study using two different tumor types in two different mouse strains, prior immunity to HSV did not inhibit the therapeutic effect of intraneoplastic inoculation. Moreover, in a strategy analogous to a vaccine booster regimen, multiple inoculations over several weeks increased the cure rate in a colon cancer model from $0 \%$ to $80 \%(24)$.

Taken together, these studies suggest that prior immunity to HSV may alter the level of gene transfer and expression within a tumor but does not alter the overall antitumor therapeutic effects of HSV following intraneoplastic tumor therapy. Indeed, immune responses may enhance treatment efficacy of oncolytic HSV therapy by generating antitumor immunity. As a corollary, immunosuppression would be predicted to reduce the overall cure rate. This possibility is of great practical interest, since patients with tumors are often spontaneously anergic or may be administered corticosteroids or other immunosuppressive agents. In one study, corticosteroids did not affect the oncolytic capacity of HSV
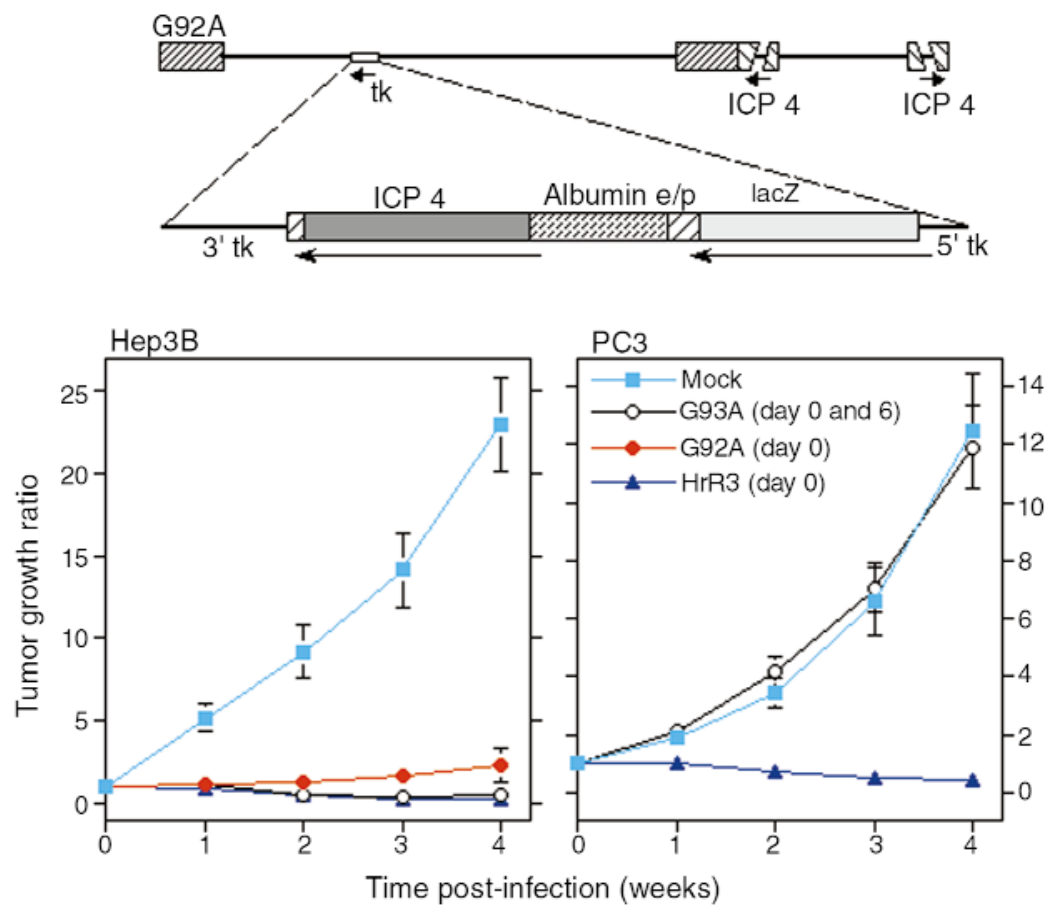

Figure 2

Targeting specific cancer cell types (reproduced from references 29 and 30 with permission). G92A expresses ICP4 under the control of an albumin promoter/enhancer sequence and preferentially replicates within albumin expressing cells. Albumin expressing human hepatomas grown in athymic mice are effectively treated by either one or two doses of G92A (left) whereas albumin non-expressing human prostate cancer cells are unaffected (right). In contrast, hrR3, an ICP6-deficient vector that is not promoter regulated is effective against either tumor type. 
in culture but did suppress immune responses to HSV and to the tumor, and they reduced the overall number of tumor cures in a syngeneic mouse tumor model (20).

\section{Promoting systemic and long-term antitumor immunity}

Using a syngeneic mouse colon cancer model, Toda et al. (25) demonstrated that oncolytic HSV growing within a tumor elicits an immune response not only to the HSV but also to specific tumor antigens. In this initial study, tumors were grown on both the right and the left flanks of syngeneic immunocompetent mice, and one of the established tumors was then treated intraneoplastically. The injected tumor promptly regressed, responding more efficiently than would typically be seen with tumors grown in athymic animals. Most importantly, however, the tumor on the opposite flank also showed regression, although the virus had not spread systemically to the opposite tumor. Rather, a CD8 ${ }^{+} \mathrm{T}$ cell-mediated response had been generated to a specific tumor cell antigen on the colon cancer cells. The specificity of this response was evident because only cells of the tumor type used were killed by splenocytes from the treated animals, and exposure to the specific tumor cell antigen rendered other cells subject to killing by these splenocytes.

These observations raise the possibility that treating local tumors will have the beneficial side effect of suppressing growth of distant metastatic tumors. Further studies (19) demonstrated that intraneoplastic infusion of HSV vectors also protected animals from delayed rechallenge with tumor, suggesting that these vectors could be developed as anti-tumor vaccines. For this "in situ vaccination" strategy, it may prove relatively straightforward to create vials of stable vector that can be used for multiple patients to induce immunity against specific tumor antigens, an attractive alternative to other tumor vaccine strategies that involve isolating cells from individual patients, altering these cells in culture, and re-inoculating them into the patient.

The recognition that conditionally replicating HSV vectors induce anti-tumor immune responses may inspire novel vector designs with improved efficacy against tumors. In particular, HSV may be engineered to carry genes for cytokines that potentiate the immune response. HSV is a versatile vector in this regard: Cytokine genes may be inserted directly into a replication competent vector backbone: alternatively, one can use helper viruses to package constructs in which the bulk of the HSV genome is replaced with multiple tandem copies of genes of interest, to create replicationdefective HSV amplicon vectors. For example, we have generated amplicon vectors containing genes for the 2 subunits of IL-12 (26). Mixed with replication-competent G207 and inoculated into colon tumors in a syngeneic mouse model, this IL-12 vector enhanced antitumor immune responses toward both ipsilateral and contralateral tumors. Similarly, recombinant replication competent HSV vectors carrying the IL-4 cDNA improve overall tumor therapy (27). This promising approach could clearly be attempted with many cytokines genes, and best of these options has yet to be identified. Moreover, the large size of HSV permits more than one such genes to be inserted into a single vector. However, because ectopic expression of cytokines could induce harmful edema, the safe use of this strategy may be limited, particularly for treating tumors in the brain.

\section{Modes of vector delivery}

While the initial development of conditionally replicating HSV vector tumor therapy focused on direct intraneoplastic instillation, more recent studies have explored other methods of delivery. Local instillation of these vectors into the peritoneum to treat ovarian cancer (16) and into the bladder to treat bladder cancer (T. Yazaki, personal communication) can cause tumor destruction without harm to surrounding normal tissues. These vectors are also effective following local vascular perfusion, as seen in a metastatic colon cancer model (15) and a model of malignant glioma (28). In both studies, vascular delivery reached multiple tumors in the perfused organ, thus extending this form of therapy from the simple instillation directly into a single tumor. Work with the glioma model showed that complement and immunoglobulins in the serum can inactivate HSV in both naive and previously treated animals, which may can limit the efficacy of intra-arterial delivery unless their effects can be blunted (28).

HSV may also be delivered intravenously to treat systemic tumors (14). Following tail vein inoculation of G207, viral gene expression is seen in multiple organs for approximately four days, but the virus does not cause overt toxicity. Intravenously administered vector also induced regression and cure of distant tumors in 2 independent metastatic prostate cancer models, and several other laboratories (T. Yazaki and F. Tufaro, personal communications) have made similarly encouraging observations on other tumor types. No deleterious vascular or systemic toxicity from the HSV vectors has yet been seen, raising the hope that HSV-based vectors could be administered systemically to treat metastatic cancer. However, this area is still in its early stages of development and many of the same issues that were previously addressed for intracerebral studies will now need to be addressed for intravenous administration. These should include studies of toxicity in several relevant animal models as well as effects of pre-immunity and possible immune effects in normal tissues.

\section{Increasing the cell specificity of HSV vectors}

By deleting the genes for TK, RR, or ICP34.5 singly or in combination, the above strategies of vector construction selectively target features that are common to many cancers. However, if viral replication can be confined to specific types of cancer cells, it should be possible to use less attenuated, and presumably more efficacious, vectors. To this end, viruses have been engineered to express altered surface glycoproteins or to employ novel receptors, antibody fragments, or other macromolecules for host cell recognition. Another appealing method of targeting viral replication to specific cells is to place an immediate-early gene essential for viral replication under the control of a tissue-specific promoter or enhancer. Thus, in the G92A vector, the albumin enhancer/promoter sequence has been used to drive 
expression of the HSV immediate-early gene encoding ICP4. ICP4-null mutant strains are incapable of replication, so G92A would be expected to replicate only in cells carrying the transcription factors that drive albumin expression. Indeed, this vector replicated > 1000-fold more efficiently in albumin-expressing hepatoma cells than in other tumor or non-tumor cells (29), and it proved effective in vivo against an albumin-expressing hepatoma but not against prostate cancer (30) (Figure 2 ). This strategy should allow HSV-based vectors to be directed against various tumor cell types, using cancer cell-specific promoter or enhancer sequences

\section{Summary and future directions}

All currently used chemotherapeutic agents have their maximal dose at the time of administration and thereafter decline in concentration. The possibility of developing an anticancer therapy whose activity can increase with time while retaining tumor-specificity is a new and uncharted area of cancer therapy. For now, it can be said that conditionally replicating vectors based on herpes simplex can be applied safely even for direct inoculation into the most HSV-sensitive organ, the brain. This reassurance opens the possibility of exploring HSV vectors for the treatment of many of the common cancers afflicting humankind. Moreover, preclinical studies thus far suggest that they are both safe and efficacious following intravascular delivery and can be effectively used in conjunction with chemotherapy, radiotherapy, and immunotherapy. Although initial studies by their very nature emphasized safety in the construction and testing of vectors, future vectors are already in hand that may improve their efficacy. It will be important to maintain a cautious attitude in developing these tools. The safety of the patient being treated is of utmost concern, but the safety of family members of the patient, of scientists, physicians and other health-care workers, as well as of the general populace and the environment must also be addressed. In safeguarding the patient, appropriate preclinical studies in virus-sensitive animals must be performed, preferably in more than one species. As new vectors or new administration routes are considered, the physician caring for the patient must always ask if a safer vector or mode of therapy could be contrived. Methods of vector delivery and of patient care must minimize problems of contamination of others in proximity to the patient. For vector construction and preparation, alternative producer cells and methods of preparation should be explored to minimize the risk of contamination with unwanted biologic agents. Finally, in vector construction, it must be remembered that these are replicating vectors and, while certain gene insertions may be therapeutically beneficial, vectors must be designed so that they would not confer new pathogenicity to the virus if it were to reach the general population.

My group has been very cautious in our initial development, pre-clinical, and clinical testing of G207. Some have criticized us for being overly cautious. But the public appropriately demands this, and, in the development of more efficacious vectors, safety must always be an important consideration and must override the competing commercial and scientific issues. Cancer is a com- mon problem in our society and we are all at risk during our lifetimes. The cancer patient and the general public recognize that risks must be taken if we are to cure cancer, but they ask that these risks be reasonable and be openly discussed.

On a personal note, my method for weighing the risk of proceeding with a clinical trial is very straightforward. I have had cancer and although I have been considered to be cured, I recognize that there is always a risk of recurrence. Ironically, I could face being treated with a vector I helped create. This not only adds energy and importance to this research but also provides me with an easy, though admittedly biased, test as to whether or not to proceed with the clinical testing of a vector: Would I allow myself to be injected with it?

Conditionally replicating herpes vectors are an exciting and promising area of cancer therapy with great future promise. I am convinced that studies can be designed that not only have the possibility for efficacy but that are also appropriately cautious to allow this novel avenue of therapy to explore its full potential in cancer care.

\section{Acknowledgments and disclaimers}

This review is by its nature a historical perspective through the author's eyes of the early development of this field. Space limitations preclude this from being an exhaustive review and this unfortunately limits appropriate recognition of many of my colleagues and of scientists worldwide who have contributed immeasurably to the development of this field. However, I specifically wish to thank S. Rabkin for his scientific contributions, advice and editorial assistance. R.L. Martuza is a member of the Scientific Advisory Board of Neurovir Inc. and has grants from and holds stock options in the company, and is supported in part by grants from the National Institutes of Health (NS32677), Department of Defense (PCRP-97 IDEA), CaPCURE, (Santa Monica, California, USA) and NF, Inc. (Massachusetts, USA).

1. Roizman, B. 1996. The function of herpes simplex virus genes: a primer for genetic engineering of novel vectors. Proc. Natl. Acad. Sci. USA. 93:11307-11312.

2. Goldstein, D.J., and Weller, S.K. 1988. Herpes simplex virus 1-induced ribonucleotide reductase activity is dispensable for virus growth and DNA synthesis: isolation and characterization of an ICP6 lacZ insertion mutant. J. Virol. 62:196-205.

3. Chou, J., Kern, E.R., Whitley, R.J., and Roizman, B. 1990. Mapping of herpes simplex virus- 1 neurovirulence to the $\gamma_{1} 34.5$ gene, a gene nonessential for growth in culture. Science. 250:1262-1266.

4. Martuza, R.L., Malick, A., Markert, J.M., Ruffner, K.L., and Coen, D.M. 1991. Experimental therapy of human glioma by means of a genetically engineered virus mutant. Science. 252:854-856.

5. Markert, J.M., Malick, A., Coen, D.M., and Martuza, R.L. 1993. Reduction and elimination of encephalitis in an experimental glioma therapy model using attenuated herpes simplex mutants that retain susceptibility to acyclovir. Neurosurgery. 32:597-603.

6. Mineta, T., Rabkin, S.D., and Martuza, R.L. 1994. Treatment of malignant gliomas using ganciclovir-hypersensitive, ribonucleotide reductasedeficient herpes simplex viral mutant. Cancer Res. 54:3963-3966.

7. Boviatsis, E., et al. 1994. Antitimor activity and reporter gene transfer into rat brain neoplasms inoculated with herpes simplex virus vectors defective in thymidine kinase or ribonucleotide reductase. Gene Ther. 1:323-331.

8. Mineta, T., Yazaki, T., Hunter, W., Rabkin, S.D., and Martuza, R.L. 1995. Attenuated multi-mutated herpes simplex virus-1 for the treatment of malignant gliomas. Nat. Med. 1:938-943.

9. Hunter, W.D., et al. 1999. Attenuated, replication-competent, herpes simplex virus type-1 mutant G207: Safety evaluation of intracerebral injection in non-human primates. J. Virol. 73:6319-6326. 
10. Markert, J., Medlock, M., Martuza, R.L., Rabkin, S., and Hunter, W. 1998. Initial report of a phase I trial of genetically engineered HSV-1 in patients with malignant glioma. In 23rd international herpesvirus workshop. York, UK. A384.

11. Lasner, T.M., et al. 1998. Toxicity and neuronal infection of a HSV-1 ICP34.5 mutant in nude mice. J. Neurovirol. 3:100-105.

12. Brown, S., et al. 1998. A Phase I dose escalating trial of intratumoral injection with ICP34.5 negative HSV1 into recurrent malignant glioma. In 23rd international herpesvirus workshop.York, UK. A386.

13. Toda, M., Rabkin, S.D., and Martuza, R.L. 1998. Treatment of human breast cancer in a brain metastatic model by G207, a replication-competent multimutated herpes simplex virus 1. Hum. Gene Ther. 9:2177-2185.

14. Walker, J.R., et al. 1999. Local and systemic therapy of human prostate cancer with the conditionally replicating herpes simplex virus vector G207. Hum. Gene Ther. 10:2237-2245.

15. Kooby, D.A., et al. 1999. Oncolytic viral therapy for human colorectal cancer and liver metastases using a multi-mutated herpes simplex virus type-1 (G207). FASEB J. 13:1325-1334.

16. Coukos, et al. 1999. Multi-attenuated herpes simplex virus-1 mutant G207 exerts cytotoxicity against epithelial ovarian cancer but not normal mesothelium, and is suitable for intraperitoneal oncolytic therapy. Cancer Gene Ther. In press.

17. Randazzo, B.P., Bhat, M.G., Kesari, S., Fraser, N.W., and Brown, S.M. 1997. Treatment of experimental subcutaneous human melanoma with a replication-restricted herpes simplex virus mutant. J. Invest. Dermatol. 108:933-937.

18. Chalavi, A., Todo, T., Martuza, R.L., and Rabkin, S.D. 1999. Replicationcompetent herpes simplex virus vector G207/cisplatin combination therapy for head and neck squamous cell carcinoma. Neoplasia. 1:162-169.

19. Todo, T., et al. 1999. Systemic antitumor immunity in experimental brain tumor therapy using a multimutated, replication-competent herpes simplex virus. Hum. Gene Ther. 10:2741-2755.

20. Todo, T., Rabkin, S.D., Chalavi, A., and Martuza, R.L. 1999. Corticos- teroid administration does not affect viral oncolytic activity, but inhibits antitumor immunity in replication-competent herpes simplex virus tumor therapy. Hum. Gene Ther. 10:2869-2878.

21. Advani, S.J., et al. 1998. Enhancement of replication of genetically engineered herpes viruses by ionizing radiation: a new paradigm for the destruction of therapeutically intractable tumors. Gene Ther. 5:160-165.

22. Advani, S.J., et al. 1999. Replication-competent, non-neuroinvasive genetically engineered herpes virus is highly effective in the treatment of therapy-resistant experimental human tumors. Cancer Res. 59:2055-2058.

23. Herrlinger, U., et al. 1998. Pre-existing herpes simplex virus-1 (HSV-1) immunity decreases, but does not abolish, gene transfer to experimental brain tumors by a HSV-1 vector. Gene Ther. 5:809-819.

24. Chalavi, A., Rabkin, S.D., Todo, T., Sundaresan, P., and Martuza, R.L. 199. Effect of prior exposure to HSV-1 on viral vector mediated tumor therapy in immunocompetent mice. Gene Ther. 6:1751-1758.

25. Toda, M., Rabkin, S.D., Kojima, H., and Martuza, R.L. 1999. Herpes simplex virus as an in situ cancer vaccine for the induction of specific antitumor immunity. Hum. Gene Ther. 10:385-393.

26. Toda, M., Martuza, R.L., Kojima, H., and Rabkin, S.D. 1998. In situ cancer vaccination: an IL-12 defective vector/replication-competent herpes simplex virus combination induces local and systemic antitumor activity. J. Immunol. 160:4457-4464.

27. Andreansky, S., et al. 1988. Treatment of intracranial gliomas in immunocompetent mice using herpes simplex viruses that express murine interleukins. Gene Ther. 5:121-130.

28. Ikeda, K., et al. 1999.. Oncolytic virus therapy of multiple tumors in the brain requires suppression of innate and elicited anti-viral responses. Nat. Med. 5:881-887.

29. Miyatake, S-I., Iyer, A., Martuza, R.L., Rabkin, S.D. 1997. Transcriptional targeting of herpes simplex virus for cell-specific replication. J. Virol. 71:5124-5132,

30. Miyatake, S-I., et al. 1999. Hepatoma-specific anti-tumor activity of an albumin enhancer/promoter regulated herpes simplex virus in vivo. Gene Ther. 6:564-572. 